Research Article

\title{
Retinal Vessel Segmentation by Deep Residual Learning with Wide Activation
}

\author{
Yuliang Ma $\mathbb{D},{ }^{1,2}$ Xue Li, ${ }^{1}$ Xiaopeng Duan, ${ }^{1}$ Yun Peng, ${ }^{3}$ and Yingchun Zhang $\mathbb{D}^{3}$ \\ ${ }^{1}$ Institute of Intelligent Control and Robotics, Hangzhou Dianzi University, Hangzhou 310018, Zhejiang, China \\ ${ }^{2}$ Key Laboratory of Brain Machine Collaborative Intelligence of Zhejiang Province, Hangzhou 310018, Zhejiang, China \\ ${ }^{3}$ Department of Biomedical Engineering, University of Houston, Houston 77204, TX, USA \\ Correspondence should be addressed to Yuliang Ma; mayuliang@hdu.edu.cn and Yingchun Zhang; yzhang94@uh.edu
}

Received 14 June 2020; Revised 14 September 2020; Accepted 21 September 2020; Published 10 October 2020

Academic Editor: Reinoud Maex

Copyright (C) 2020 Yuliang Ma et al. This is an open access article distributed under the Creative Commons Attribution License, which permits unrestricted use, distribution, and reproduction in any medium, provided the original work is properly cited.

Purpose. Retinal blood vessel image segmentation is an important step in ophthalmological analysis. However, it is difficult to segment small vessels accurately because of low contrast and complex feature information of blood vessels. The objective of this study is to develop an improved retinal blood vessel segmentation structure (WA-Net) to overcome these challenges. Methods. This paper mainly focuses on the width of deep learning. The channels of the ResNet block were broadened to propagate more lowlevel features, and the identity mapping pathway was slimmed to maintain parameter complexity. A residual atrous spatial pyramid module was used to capture the retinal vessels at various scales. We applied weight normalization to eliminate the impacts of the mini-batch and improve segmentation accuracy. The experiments were performed on the DRIVE and STARE datasets. To show the generalizability of WA-Net, we performed cross-training between datasets. Results. The global accuracy and specificity within datasets were $95.66 \%$ and $96.45 \%$ and $98.13 \%$ and $98.71 \%$, respectively. The accuracy and area under the curve of the interdataset diverged only by $1 \% \sim 2 \%$ compared with the performance of the corresponding intradataset. Conclusion. All the results show that WA-Net extracts more detailed blood vessels and shows superior performance on retinal blood vessel segmentation tasks.

\section{Introduction}

Image segmentation in retinal blood vessels has an important medical application value [1]. Analyzing the changes of the retinal vascular structure is a key part in diagnosing retinal diseases such as diabetic retinopathy (DR) [2], hypertension, and arteriosclerosis. However, the retinal blood vessels show complex network structures and low contrast, causing difficulties in manual segmentation.

Many methods have been proposed in recent years by researchers in the field. Broadly, these methods can be divided into two groups: unsupervised and supervised approaches [3]. In unsupervised approaches, main algorithms include matched filtering [4], morphological processing [5], vascular tracking [6], and model-based [7]. However, an extreme limitation of these unsupervised methods is that they heavily rely on handcrafted features for vessel representation and segmentation. Besides, the parameters need to be elaborately designed. Therefore, unsupervised methods are inferior to supervised approaches in terms of segmentation accuracy.

Supervised learning, different from unsupervised methods, requires manual annotations to build the optimally predictive model. There are two processors which are needed: one is an extractor, and the other one is a classifier. The features of the retinal vessel can be extracted by Gabor filter [8], Gaussian filter [9], and so on. In traditional machine learning, K-nearest neighbor (K-NN), SVM, AdaBoost, etc. are often used to train the classifier [10-12]. In the study conducted by Zhu et al. [13], filters, morphological operators, and linear detection operators were used to extract retinal vascular morphological features as prior information for the classifier. Based on a fully connected conditional random field (CRF) model, Orlando et al. [14] 
used a structured output support vector machine to learn model parameters and perform retinal vessel segmentation. For these traditional supervised methods, the final results predicted are greatly influenced by the features used for classification. However, they are often defined empirically, which prevents the generalization of the unknown geometric transformation of blood vessels.

Given the aforementioned limitations, it remains a challenging task to effectively and automatically segment retinal blood vessels that warrants further studies using novel techniques. Deep learning, with powerful data representation capabilities, has been widely used in retinal image segmentation tasks. Fu et al. [15] formulated the vessel segmentation to a holistically-nested edge detection (HED) problem and utilized the fully convolutional network (FCN) to generate the vessel probability map. Mo and Zhang [16] developed a deep supervised FCN by leveraging multilevel hierarchical features. Liskowski and Krawiec [17] regarded blood vessel segmentation as a binary classification task, using convolutional neural network (CNN) to perform pixel-bypixel classification, and combined structured prediction methods to classify multiple pixels at the same time. The results obtained are better than most traditional algorithms. However, the network has tens of millions of parameters. Recently, it is more challenging to develop a simpler but effective model to accurately segment small vessels. Jin et al. [18] proposed deformable U-Net (DUNet), which integrated deformable convolution blocks, to detect the tiny vessels. It has fewer parameters but longer calculation time. Gu et al. [19] developed the context encoder network (CE-Net) to preserve spatial information for medical image segmentation. However, its segmentation accuracy on the vessel segmentation task is unsatisfactory. Azad et al. [20] took full advantages of U-Net [21], dense convolutions [22], and bidirectional ConvLSTM (BConvLSTM) [23] to segment vessels. They achieved a higher accuracy, but excessive use of dense block (DB) consumes memory greatly. Budak et al. [24], in their recent work, showed that the weak and thin vessels are better segmented by the cascading $\mathrm{CNN}$ of lesser depth as long as feature utilization is improved. For this reason, and motivated by the fact that wide activation, which focuses on the width of deep learning, propagates more low-level features with the same parameter complexity [25], in this study, we develop a retinal vessel segmentation method by deep residual learning based on wide activation.

The remaining of this paper is organized as follows: Section 2 presents the proposed method, Section 3 provides implementation details, Section 4 analyzes the experimental results, and discussion and conclusion are drawn in Section 5 and Section 6, respectively.

\section{Proposed Method}

An overview of the proposed segmentation model is shown in Figure 1. The original retinal image is preprocessed and input to WA-Net, and then the segmented image is output after network mapping. This framework has two main modules, denoted as wide activated module and LASPP module, which will be introduced in the following parts.

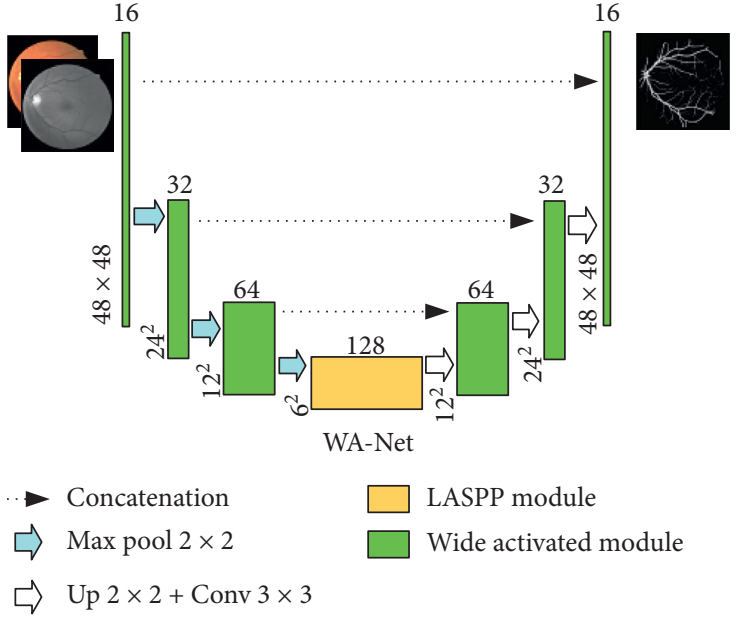

Figure 1: Retinal vessel image segmentation model. The numbers above each block stand for the input filters. At the lower left edge are the dimensions of each block. In the legend, Concatenation means the merger of channels, Max pool denotes max pooling, Up indicates upsampling, and Conv represents convolution. The number following them is the kernel size.

2.1. Wide Activated Module. In the traditional convolutional neural network, the output of the $l^{\text {th }}$ layer can be calculated by the following equation:

$$
x_{l}=H_{l}\left(x_{l-1}\right) \text {, }
$$

where $x_{l}$ denotes the output of the $l^{\text {th }}$ layer, $x_{l-1}$ denotes the output of the former $(l-1)^{\text {th }}$ layer, and $H$ denotes a convolution often activated with ReLU, where ReLU is defined as $f\left(x_{l-1}\right)=\max \left(0, x_{l-1}\right)$.

In order to make full use of features, He et al. [26] proposed residual mapping to build a deeper network. The definition of residual mapping is as follows:

$$
F\left(x_{l}\right)=Y\left(x_{l}\right)-x_{l},
$$

where $x_{l}$ is the input, $Y\left(x_{l}\right)$ is the output mapping, and $F\left(x_{l}\right)$ is the residual mapping.

Wide activated module (WDSR-A) [25] is a kind of residual module which is based on wide activation as shown in Figure 2. It mainly consists of four layers: two convolution layers, an activation layer (ReLU) and an identity layer. In the practical experiment, a $1 \times 1$ convolution is used in the identity layer when channels do not match.

2.1.1. Original Residual Block. For the original residual block (Figure 3(a)), suppose the width of the identity mapping pathway is $c_{1}$ and the width before activation in the residual block is $c_{2}$; then, $c_{2}=c_{1}$, so the parameters in each original residual block are $2 \times c_{1}^{2} \times k^{2}$, where $k$ is the kernel size.

2.1.2. Wide Activated Residual Block. Figure 3(b) shows the residual block with wide activation. It is calculated by the following equation:

$$
\widehat{c}_{2}=r \times \widehat{c}_{1},
$$




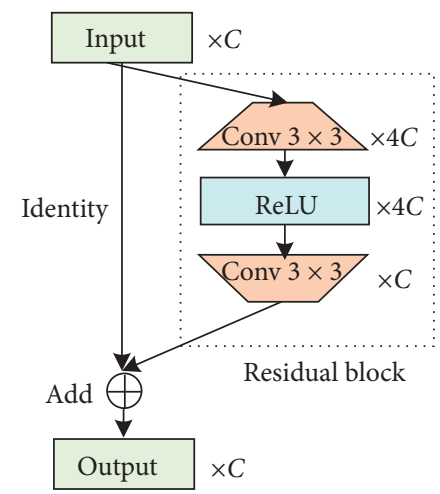

FIGURE 2: Schematic of WDSR-A. Identity represents the identity mapping layer, Add denotes the element-wise summation over channels. In the dashed box, Conv stands for convolution, ReLU indicates the activation layer, $C$ is the abbreviation of the channel, and the two conical shapes are the convolution layers that expand and slim the channel, respectively. The principle of wide activation is expanding features before the activation layer without increasing computation. A further explanation is shown in Figure 3.

where $r$ is the expansion factor before activation and $\widehat{c}_{1}$ and $\widehat{c}_{2}$ are the width of the identity mapping pathway in WDSR$A$ and the width before ReLU, respectively.

When the patch size of the input is fixed, the computational complexity is a fixed proportion of the parameters. In order to make WDSR-A maintain the same complexity as the original residual block, the following equation is established:

$$
c_{1}^{2}=\widehat{c}_{1} \times \widehat{c}_{2}=r \times \widehat{c}_{1}^{2} .
$$

According to the above equation, the width of the identity mapping pathway in WDSR-A needs to be slimmed by factor $\sqrt{r}$, and the width before activation should be expanded by $\sqrt{r}$. This paper takes $r=4$.

2.2. LASPP Module. A major challenge in vessel segmentation is how to capture tiny blood vessel features. This problem was solved by atrous convolution [27], which allows to enlarge the receptive field without increasing parameters.

The principle of the atrous convolution is to insert 0 pixels between each pixel of the traditional convolutional kernel, i. e., to increase the dilation rate $d$ of the network. For each pixel $i$ of the output $y$, the process of the atrous convolution is expressed as

$$
y(i)=\sum_{k} x(i+d \times k) w(k)
$$

where $w(k)$ denotes a filter, $k$ is the filter size, $x$ denotes the input, and $d$ is the dilation rate. As shown in Figure 4, the size of the convolution kernel is $3 \times 3$, and Figures $4(\mathrm{a})-4$ (c) correspond to atrous convolutions of $d=1, d=2$, and $d=3$, respectively.

Different dilation rates can be used to change the receptive field. For the atrous convolutional layer with the dilation rate of $d$ and kernel size of $k$, the receptive field size is calculated by the following equation::

$$
R_{f}=(k-1) \times(d-1)+k .
$$

For example, if a convolutional kernel size is $3 \times 3$ with dilation rate $d=3$, the corresponding receptive field size is 7 . The superposition of multiple convolutional layers allows for a greater receptive field.

Atrous spatial pyramid pooling (ASPP) [28] is a model based on the atrous convolution. It adopts several parallel convolutional layers with different expansion rates to improve the segmentation performance. Inspired by this, at the bottom of the network, we designed a module like ASPP (LASPP) to preserve multiscale features of blood vessels, as shown in Figure 5. The dilation rates used in the four convolutional layers are $d=2^{i}, i=0,1,2,3$, with kernel size $3 \times 3$ and activated by ReLU. Finally, the features extracted with different dilation rates are added to generate the fusion result and send to the decoding structure.

2.3. Weight Normalization. In training deep neural networks, batch normalization (BN) is frequently used after each convolution to solve the internal covariate shift problem [29]. However, BN has the drawback of data dependence on the mini-batch [25]. In this way, we prefer to use weight normalization (WN) to speed up the convergence speed of the network and improve the accuracy of training and testing [30].

Weight normalization, in simple terms, is the reparameterization of weight vectors in the CNN. Assume the output $\mathbf{y}$ has the following form:

$$
\mathbf{y}=\mathbf{w} \cdot \mathbf{x}+b,
$$

where $\mathbf{w}$ is a $k$-dimensional weight vector, $b$ denotes a scalar bias term, and $\mathbf{x}$ is a $k$-dimensional vector of the input features. WN reparameterizes the weight vectors with new parameters by the following equation::

$$
\mathbf{w}=\frac{g}{\|\mathbf{N}\|} \mathbf{N}
$$

where $\mathbf{N}$ is a $k$-dimensional vector, $g$ denotes a scalar, and $\|\mathbf{N}\|$ is the Euclidean norm of $\mathbf{N}$. With this formalization, we will have $\|\mathbf{w}\|=g$, which is independent of parameter $\mathbf{N}$ [30].

2.4. Network Structure: WA-Net. Figure 6 illustrates the wide activation network (WA-Net). The architecture consists of two parts: encoder and decoder. For the encoder part, the image patches are input to the network for batch normalization (only one time). Then, each WDSR-A module is followed by a $2 \times 2$ max pooling layer. In the decoding phase, $\mathrm{Up}+\mathrm{conv}$ means that the upsampling was followed by a $3 \times 3$ convolution without ReLU. The dashed line in Figure 6 represents a global shortcut with a convolution of $1 \times 1$ and leaky ReLU. Behind WDSR-A1 in the decoding section is another leaky ReLU. We add the outputs of the two leaky ReLUs and put them into a $1 \times 1 \times 2$ convolution layer with softmax to do classification. 


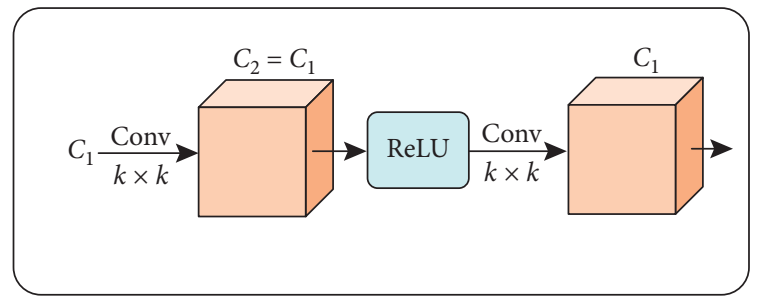

Original residual block

(a)

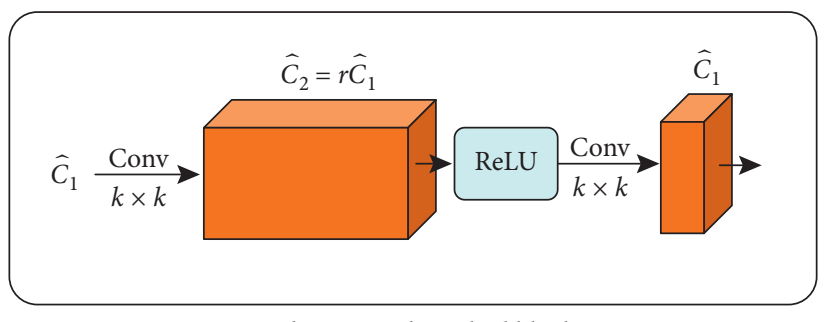

Wide activated residual block

(b)

FIGURe 3: Principle of wide activation [25]. $C_{i}$ and $\widehat{C}_{i}, i=1,2$, stand for channels, $r$ is the expansion factor, and $k \times k$ below Conv is the kernel size. In deep learning, the width refers to the number of channels. Wide activation focuses on the width (the $C$ parameter) to improve feature utilization.

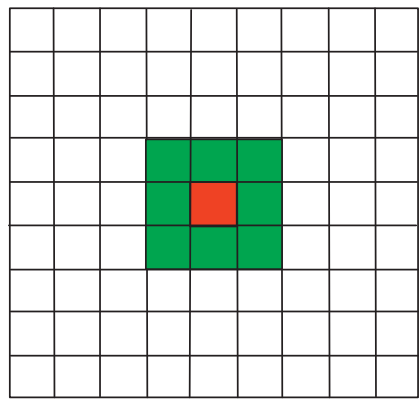

(a)

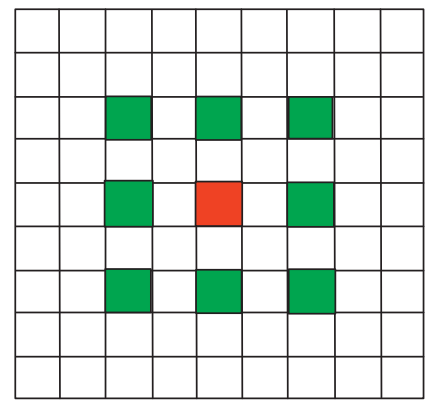

(b)

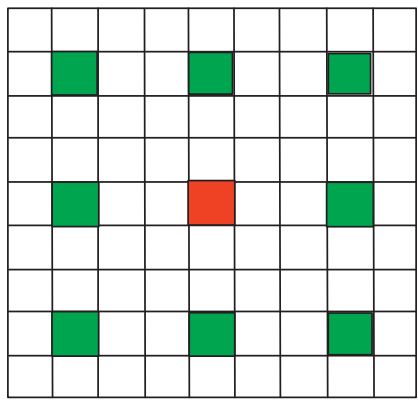

(c)

Figure 4: Schematic of atrous convolutions. (a) $d=1$. (b) $d=2$. (c) $d=3$.

The motivation of leaky ReLU is to avoid zero gradients. It is defined as follows:

$$
f\left(x_{i}\right)= \begin{cases}x_{i}, & \text { if } x_{i}>0 \\ a_{i} x_{i}, & \text { if } x_{i} \leq 0\end{cases}
$$

where $x_{i}$ is the input on the $i^{\text {th }}$ channel and $a_{i}$ is a coefficient controlling the slope of the negative part. It degenerates into $\operatorname{ReLU}$ when $a_{i}=0$. This paper sets $a_{i}=0.3$ for leaky ReLU. The specific parameter settings of each module are shown in Table 1.

2.5. Loss Function. In retinal images, the distribution of blood vessels and non-blood vessels is unbalanced. In order to solve this problem, a combined loss function is adopted. It can be defined by the following equation:

$$
L(\bar{y}, y)=L_{\mathrm{CE}}(\bar{y}, y)+L_{\mathrm{DICE}}(\bar{y}, y)
$$

where $L_{\mathrm{CE}}(\bar{y}, y)$ denotes cross-entropy, which is defined as follows:

$$
L_{\mathrm{CE}}(\bar{y}, y)=-\sum_{i}\left(y_{i} \log \overline{y_{i}}+\left(1-y_{i}\right) \log \left(1-\overline{y_{i}}\right)\right) .
$$

And $L_{\mathrm{DICE}}(\bar{y}, y)$ denotes a loss function based on the dice coefficient [31]. It is defined by the following equation:

$$
L_{\mathrm{DICE}}(\bar{y}, y)=1-\frac{2 \sum_{i} y_{i} \overline{y_{i}}+k}{\sum_{i} y_{i}+\sum_{i} \overline{y_{i}}+k},
$$

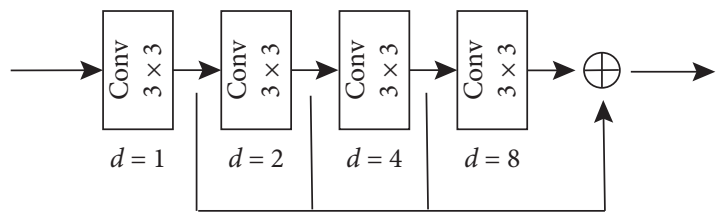

Figure 5: Schematic of the LASPP module.

where $i$ is the number of pixels, $y_{i}$ is the ground truth, and $\overline{y_{i}}$ is the predicted result, respectively. $k$ is a smooth value set to 1.0 to correct the function.

\section{Implementation}

The network model was implemented under PyCharm simulation platform using Python 3.6 with Tensorflow1.13. All experiments were conducted in a 64 bit Windows 10 laptop with Intel Core i7-8750H CPU @ $2.20 \mathrm{GHz} 2.21 \mathrm{GHz}$, 16 GB RAM, NVIDIA GeForce GTX 1050Ti GPU.

3.1. Data and Data Preprocessing. The fundus images used in the experiment are from two public datasets: DRIVE (Digital Retinal Images for Vessel Extraction) [10] and STARE (Structured Analysis of the Retina) [32]. There are 40 images in the DRIVE dataset with a resolution of $565 \times 584$, while the STARE dataset consists of 20 images with a pixel size of $605 \times 700$. Experts' manual labels in both datasets are available as the ground truth. 


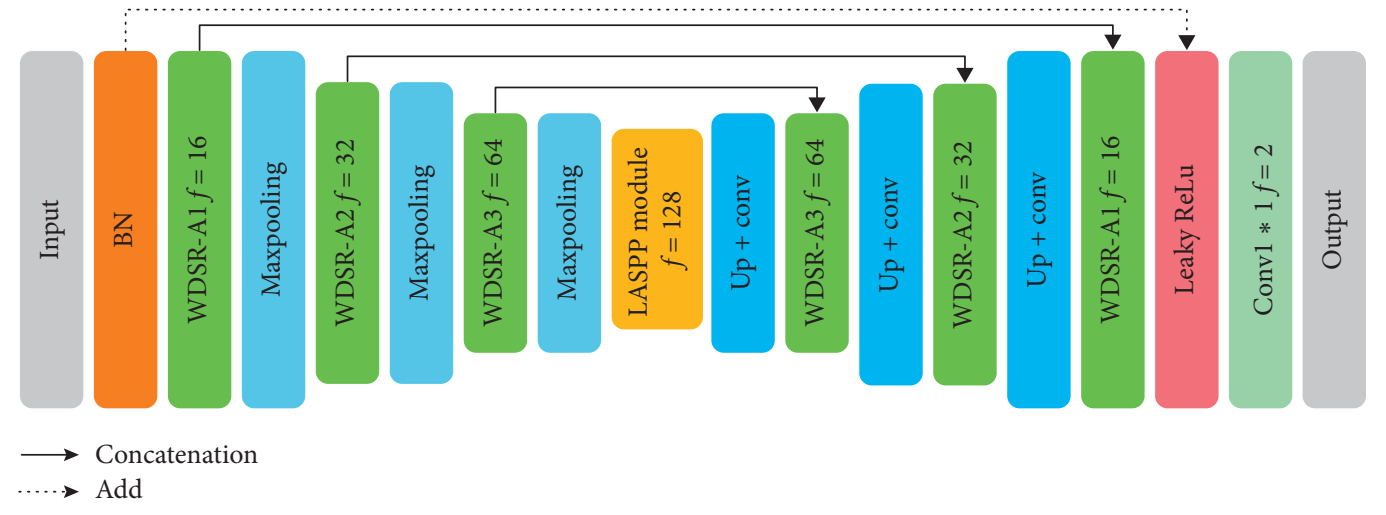

Figure 6: WA-Net structure.

Table 1: Modules designed in WA-Net.

\begin{tabular}{lccc}
\hline WDSR-A1 & WDSR-A2 & WDSR-A3 & LASPP \\
\hline $3 \times 3 \times 64$ & $3 \times 3 \times 128$ & $3 \times 3 \times 256$ & $3 \times 3 \times 128$, ReLU, $d=1$ \\
ReLU & ReLU & ReLU & $3 \times 3 \times 128$, ReLU, $d=2$ \\
$3 \times 3 \times 16$ & $3 \times 3 \times 32$ & $3 \times 3 \times 64$ & $3 \times 3 \times 128$, ReLU, $d=4$ \\
$1 \times 1 \times 16$ & $1 \times 1 \times 32$ & $1 \times 1 \times 64$ & $3 \times 3 \times 128$, ReLU, $d=8$ \\
\hline
\end{tabular}

In the deep convolutional neural network, appropriate preprocessing allows better segmentation of retinal blood vessels. In this study, all images were preprocessed as follows: firstly, the RGB images were converted into grayscale and standardized. Then, contrast-limited adaptive histogram equalization (CLAHE), which is a method of image enhancement, was used to improve the brightness and contrast of the image [33]. To further improve image quality, we introduced gamma correction, where $\gamma=1.2$. The preprocessed images were divided into local overlapping patches by the sliding window with $48 \times 48$ and the stride size of 5 . The patches sampled randomly and preprocessed are shown in Figure 7.

3.2. Training Details. Adam [34] is a common optimizer that speeds up network convergence. The proposed network selected Adam as the optimizer with initial learning rate 0.001 , and $\beta_{1}=0.9, \beta_{2}=0.999$, and $\varepsilon=10^{-8}$. The batch size and epochs were set to 32 and 100 , respectively. A kernel weight initialization method named as He normal, which is proposed by $\mathrm{He}$ et al. [35], was used to initialize the kernel weights of the WDSR-A modules.

As for the division of datasets, the DRIVE dataset has been divided into the training set (20 images) and testing set (the rest 20 images) fixedly. Considering that the STARE dataset does not explicitly provide the training and testing sets, this paper selects the first 10 images as training images and the rest 10 as test images according to Wang et al. [36]. In this study, we used the patch-based strategy to reduce overfitting. During the training process, the DRIVE and STARE datasets were divided into 160,000 and 200,000 patches, respectively, of which $90 \%$ patches were selected for training, and the other $10 \%$ were used as validation.
3.3. Performance Evaluation. In order to quantitatively evaluate the blood vessel segmentation effect, 5 evaluation metrics were used, including accuracy (Acc), sensitivity (Sens), specificity (Spec), F1 score (F1), and area under the curve (AUC). The first 4 evaluation indicators are defined as

$$
\begin{aligned}
\text { Acc } & =\frac{T_{P}+T_{N}}{T_{P}+F_{P}+T_{N}+F_{N}}, \\
\text { Sens } & =\frac{T_{P}}{T_{P}+F_{N}}, \\
\text { Spec } & =\frac{T_{N}}{T_{N}+F_{P}}, \\
\text { Prec } & =\frac{T_{P}}{T_{P}+F_{P}}, \\
F 1 & =2 \times \text { Prec } \times \frac{\text { Sens }}{\text { Prec }+ \text { Sens }},
\end{aligned}
$$

where $T_{P}$ is true positive, indicating the correctly segmented vascular pixels, $T_{N}$ is true negative, standing for the correctly segmented nonvascular pixels, $F_{P}$ is false positive, denoting incorrectly segmented vascular pixels, and $F_{N}$ is false negative, denoting incorrectly segmented nonvascular pixels.

The receiver operating characteristic (ROC) curve is also an important curve to measure the effect of retinal blood vessel segmentation. The closer the area under the curve (AUC) is to 1 , the more accurate a model is.

\section{Experimental Results}

4.1. Vessel Segmentation Results. The proposed WA-Net was compared to custom U-Net on DRIVE and STARE datasets. Figures 8 and 9 are the segmentation results. In these figures, the first row shows healthy retinal images, while the second shows unhealthy retinal images. As can be seen from Figures 8 and 9, the segmentation results were consistent with those of experts and even better on some small vascular branches. In addition, the noise and erroneous segmentation were reduced. Furthermore, WA-Net obtained desirable segmentation results in those weak vessels. On the DRIVE 


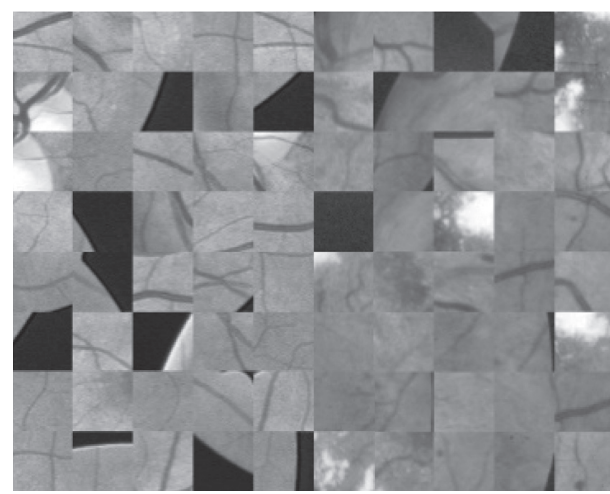

(a)

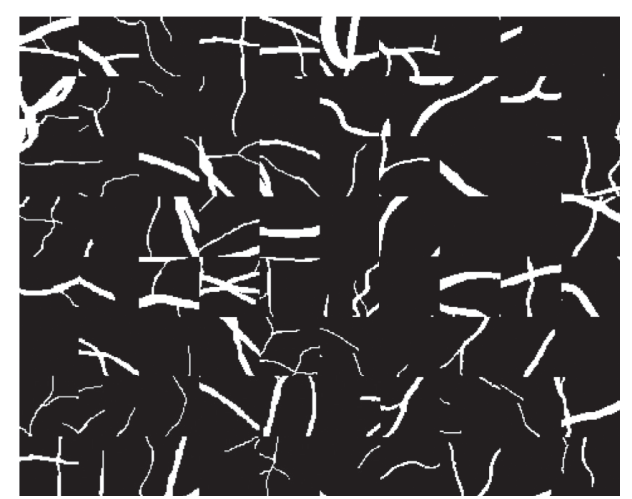

(b)

FIgURE 7: Patches sampled for model training: (a) the preprocessed patches; (b) the corresponding ground truth.
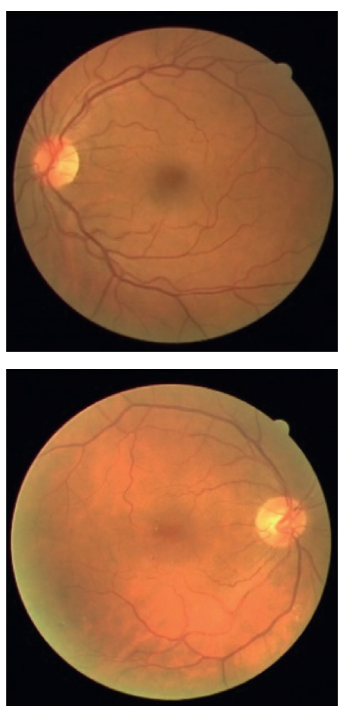

(a)
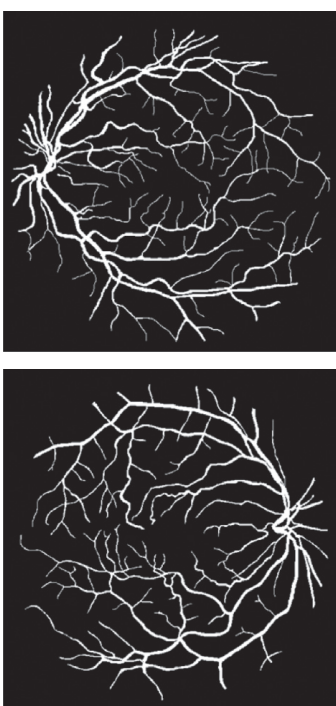

(b)
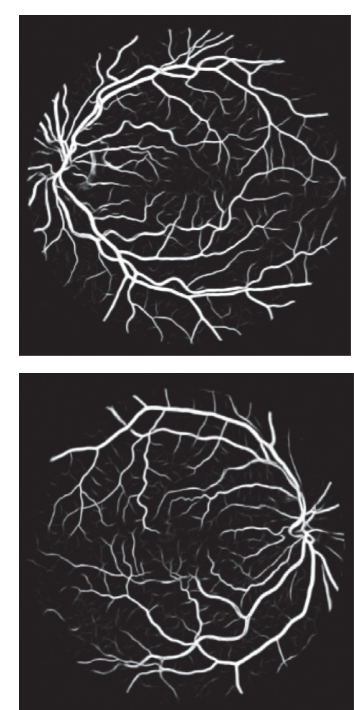

(c)
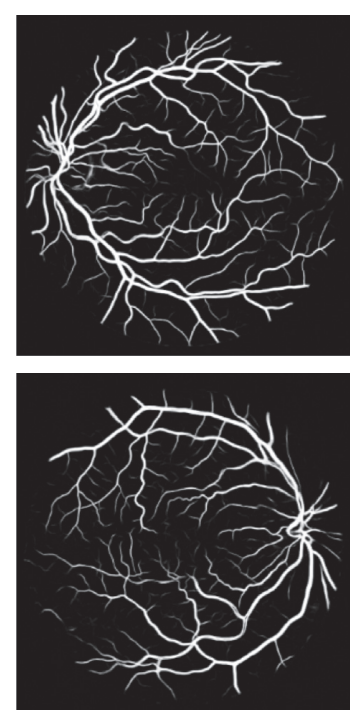

(d)

FIGURE 8: Comparison of the segmentation results on the DRIVE dataset: (a) original image; (b) ground truth; (c) U-Net; (d) proposed WANet.
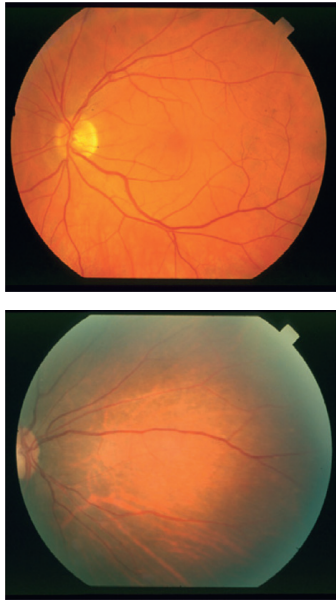

(a)
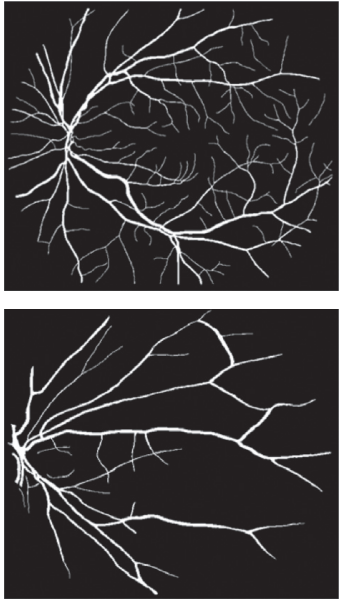

(b)
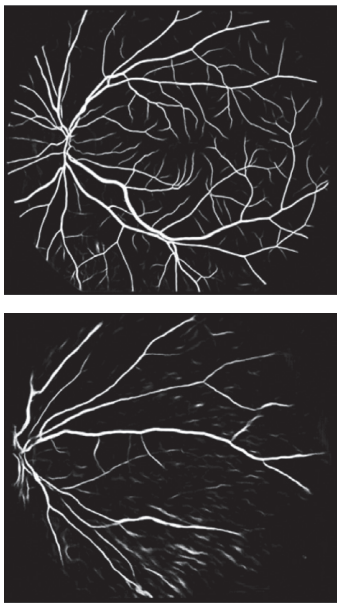

(c)
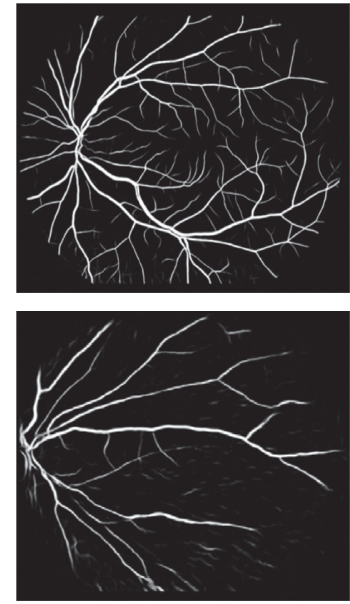

(d)

FIGURE 9: Comparison of the segmentation results on the STARE dataset: (a) original image; (b) ground truth; (c) U-Net; (d) proposed WANet. 
dataset, WA-Net had fewer segmentation errors and enhanced for some small vessel segmentation. On the STARE dataset, it is worth noting that the segmentation errors were significantly reduced. On the other hand, for the healthy group, WA-Net had less vascular noise and more continuity. For the unhealthy group, U-Net was more prone to mis-segmentation. These results proved that the proposed network had a better segmentation than U-Net.

4.2. Comparison with Other Methods. In order to further verify the performance of WA-Net in retinal blood vessel segmentation, this paper compared the evaluation metrics with existing methods on DRIVE and STARE datasets, respectively. Tables 2 and 3 present the global performance of the methods used in different literature studies on the test set.

Generally speaking, the performance of unsupervised methods is not as effective as supervised approaches. As can be seen from Table 2, on the DRIVE dataset, F1, Sens, Spec, Acc, and AUC of WA-Net were 0.8222, 0.7875, 0.9813, 0.9566 , and 0.9794 , respectively, which were superior to most algorithms. $F 1$ obtained the highest value, and Sens was only slightly lower than CE-Net. For Acc and AUC, DCCMED-Net and WA-Net achieved the best results, which further verify the effectiveness of increasing feature utilization. Acc of WA-Net was slightly lower than that of DCCMED-Net due to the latter network cascaded three encoder-decoder modules, while WA-Net used one. However, DCCMED-Net in Sens was dramatically lower than WA-Net $6.11 \%$. This phenomenon also appeared on WANet of Table 3. A possible explanation might be the extreme imbalance of blood vessels and nonvessels. In retinal images, the pixels of the blood vessels only occupy a small proportion of the entire image. When they achieve a high global accuracy, a small number of mis-segmented pixels will have a greater impact on blood vessel pixels but less impact on background pixels. Therefore, the sensitivity is relatively low.

As shown in Table 3, Spec and Acc of WA-Net achieved the highest on the STARE dataset. Dense U-Net outperformed WA-Net 1.74\% in Sens, but Spec, Acc, and AUC of WA-Net were $1.49 \%, 1.07 \%$, and $1.21 \%$ higher than Dense U-Net. To sum up, by comparing all the listed results, WANet obtained competitive performances on DRIVE and STARE datasets.

4.3. Segmentation Results between Datasets. In our work, we proved the generalization ability of the proposed network. Considering the DRIVE and STARE datasets were obtained using two devices with obviously different physical resolutions, this paper verified a more demanding scenario where one dataset was used for training and another for testing. Verification was performed on both DRIVE and STARE datasets, with the verification curve shown in Figure 10.

The tested AUC and Acc indicators of cross-training are summarized in Table 4. As listed in Table 4, when tested on the STARE dataset, we obtained the highest AUC, while Acc was lower than Yan et al. [42] slightly. However, Acc and AUC tested on the DRIVE dataset were undesirable. One possible explanation might be that the DRIVE dataset comprises many thin blood vessels, while the training set (STARE) mainly contains thick blood vessels [18]. On the other hand, we compared the performance within datasets (Tables 2 and 3) and found that the performance of Acc and AUC between datasets diverged only 1\% 2\% compared with the intradatasets. All these results demonstrated generalizability of WA-Net.

4.4. The Influence of Network Structures. This section investigated the effects of using the wide activation structure and atrous convolution. We adjusted WA-Net and presented the performance indicators on the DRIVE dataset. The wide activated module in WA-Net was replaced with the preactivated residual module [46]: BN-ReLU-Conv $\longrightarrow \mathrm{BN}$ ReLU-Conv, and LASPP module removed WN, named as Network_1. Based on Network_1, according to the traditional CNN, channels were set to 32-64-128-256-128-64-32, named as Network_2. Based on WA-Net, reduce the number of layers in the original LASPP module to 3 as Network_3, and then increase layers to 5 , where $d=16$ of the 5 th layer, named as Network_4. The details of these networks are presented in Figure 11. Furthermore, ROC curves of different structures are shown in Figure 12. The closer the ROC curve to the top-left border is in the ROC coordinates, the more accurate a model is.

Figure 11 shows that the thick blood vessel branches could be segmented by all adjusted networks, but WA-Net greatly reduced mis-segmentation. For complicated vessels, it is difficult for the network to separate small blood vessels when the thick and thin vessels are close to each other. At the junction of thick blood vessels, more information can be extracted by WA-Net, while Network_3 did not detect it at all. On multiconnected small blood vessels, it is difficult for the segmentation algorithms to proceed precisely due to the local lesion. In summary, WA-Net is able to distinguish different vessels and present a better performance than the other networks.

\section{Discussion}

The difficulty in segmenting retinal blood vessels accurately from RGB images mainly arises from their low pixel intensity, which makes them (especially some tiny vessels) similar to the background and results in difficult segmentation. To address this issue, we proposed WA-Net based on wide activation. With the help of the widened channels, more vessel information was transmitted to the subsequent atrous convolutional layers. Then, convolutional layers superimposed with different dilation ratios are used to capture contextual weak blood vessel information of different sizes more effectively. Due to the difficulty in recovering some low-level information, skip layer connection is utilized to directly fuse low-level information and highlevel information in the network structure. Comparison of the results obtained with several methods and the proposed algorithm on the DRIVE and STARE datasets shows that the segmentation accuracy by WA-Net has led to a higher 
TABLE 2: Comparison of the performance among different methods on the DRIVE dataset.

\begin{tabular}{|c|c|c|c|c|c|c|c|}
\hline Type & Method & Year & $F 1$ & Sens & Spec & Acc & AUC \\
\hline \multirow{3}{*}{ Unsupervised } & Roychowdhury et al. [37] & 2015 & - & 0.7395 & 0.9782 & 0.9494 & 0.9672 \\
\hline & Azzopardi et al. [38] & 2015 & -- & 0.7655 & 0.9704 & 0.9442 & 0.9614 \\
\hline & Zhang et al. [39] & 2016 & - - & 0.7743 & 0.9725 & 0.9476 & 0.9636 \\
\hline \multirow{8}{*}{ Supervised } & Li et al. [40] & 2015 & - & 0.7569 & 0.9816 & 0.9527 & 0.9738 \\
\hline & Liskowski and Krawiec[17] & 2016 & - - & 0.7763 & 0.9768 & 0.9495 & 0.9720 \\
\hline & Chen $[41]$ & 2017 & - & 0.7426 & 0.9735 & 0.9453 & 0.9516 \\
\hline & Yan et al. [42] & 2018 & - - & 0.7631 & 0.9820 & 0.9538 & 0.9750 \\
\hline & R2U-Net [43] & 2018 & 0.8171 & 0.7792 & 0.9813 & 0.9556 & 0.9784 \\
\hline & DCCMED-Net $^{\mathrm{a}}[24]$ & 2019 & -- & 0.7268 & 0.9912 & 0.9681 & 0.9819 \\
\hline & CE-Net [19] & 2019 & - - & 0.8309 & -- & 0.9545 & 0.9779 \\
\hline & WA-Net (ours) & 2020 & 0.8222 & 0.7875 & 0.9813 & 0.9566 & 0.9794 \\
\hline
\end{tabular}

${ }^{a}$ The listed result is obtained by DCCMED-Net with batch size 32 , which is the same as WA-Net.

TABLE 3: Comparison of the performance among different methods on the STARE dataset.

\begin{tabular}{|c|c|c|c|c|c|c|c|}
\hline Type & Method & Year & $F 1$ & Sens & Spec & Acc & AUC \\
\hline \multirow{3}{*}{ Unsupervised } & Roychowdhury et al. [37] & 2015 & - - & 0.7317 & 0.9842 & 0.9560 & 0.9673 \\
\hline & Azzopardi et al. [38] & 2015 & - - & 0.7716 & 0.9701 & 0.9497 & 0.9563 \\
\hline & Zhang et al. [39] & 2016 & -- & 0.7791 & 0.9758 & 0.9554 & 0.9748 \\
\hline \multirow{7}{*}{ Supervised } & Li et al. [40] & 2015 & - - & 0.7726 & 0.9844 & 0.9628 & 0.9879 \\
\hline & Liskowski and Krawiec[17] & 2016 & - - & 0.7867 & 0.9754 & 0.9566 & 0.9785 \\
\hline & Roychowdhury et al. [44] & 2016 & - & 0.7720 & 0.9730 & 0.9510 & 0.9690 \\
\hline & Chen [41] & 2017 & - - & 0.7295 & 0.9696 & 0.9449 & 0.9557 \\
\hline & Yan et al. [45] & 2018 & - - & 0.7581 & 0.9846 & 0.9612 & 0.9801 \\
\hline & Dense U-Net [36] & 2019 & - - & 0.7914 & 0.9722 & 0.9538 & 0.9704 \\
\hline & WA-Net (ours) & 2020 & 0.8223 & 0.7740 & 0.9871 & 0.9645 & 0.9825 \\
\hline
\end{tabular}

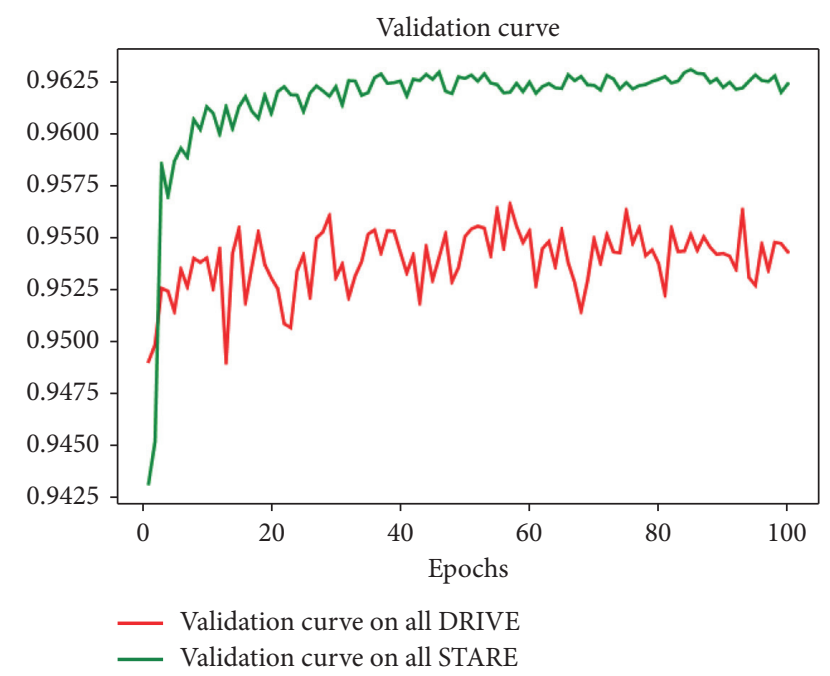

FIgURE 10: Validation curve on two datasets.

level as shown in Tables 2 and 3. In terms of the algorithm itself, it is expected that it will be robust and accurate. Therefore, cross-training between datasets is performed in Table 4, which verified the generalization performance of the model. Utilizing the characteristic that the number of feature channels is doubled at each downsampling step, the slimmed identity mapping path reduced the parameters of WA-Net.

Despite the improvement achieved in this study, there are still several limitations. Considering that the deep
TABLE 4: Comparison of the segmentation performance between datasets.

\begin{tabular}{lcccc}
\hline Dataset & Method & Year & Acc & AUC \\
\hline & Li et al. [40] & 2015 & 0.9545 & 0.9671 \\
STARE (trained on & Yan et al. [42] & 2018 & 0.9580 & 0.9678 \\
DRIVE) & DUNet [18] & 2018 & 0.9474 & 0.9571 \\
& WA-Net & 2020 & $\mathbf{0 . 9 5 6 4}$ & $\mathbf{0 . 9 7 0 7}$ \\
& Li et al. [40] & 2015 & 0.9486 & 0.9677 \\
DRIVE (trained on & Yan et al. [42] & 2018 & 0.9444 & 0.9568 \\
STARE) & DUNet [18] & 2018 & 0.9481 & 0.9718 \\
& WA-Net & 2020 & $\mathbf{0 . 9 4 7 8}$ & $\mathbf{0 . 9 7 0 0}$ \\
\hline
\end{tabular}

learning segmentation method could produce the most accurate results when there are sufficient labeled data, while fewer samples are there in retinal images, a more effective data augmentation can be achieved using the generative adversarial network (GAN). In addition, although the parameters of WA-Net are reduced, there is still a long computational time due to the introduction of weight normalization. Further investigation on how to effectively reduce the calculation time is required.

The segmentation of retinal blood vessels is the first and a critical step for automated vessel analysis. After blood vessel segmentation, more advanced analysis can be performed, for example, investigating its diagnostic and prognostic values for eye diseases such as arteriosclerosis and hypertension. Besides, in order to obtain more accurate results in medical image segmentation tasks, we plan to extend the WA-Net 

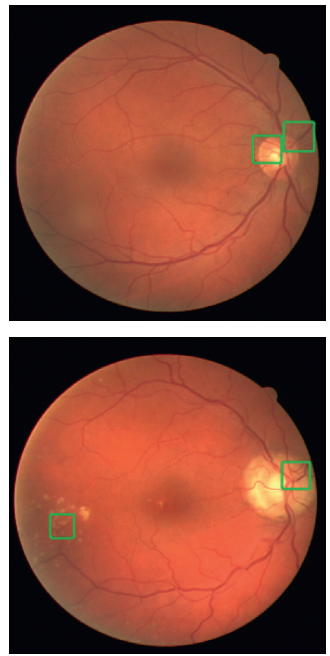

(a)
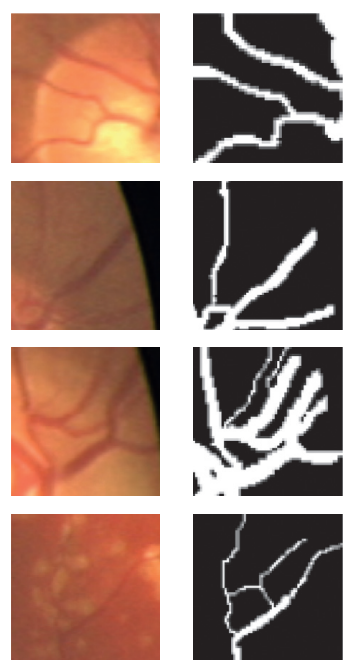

(b)

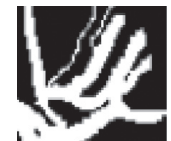

1

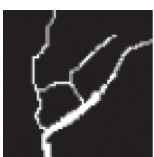

(c)
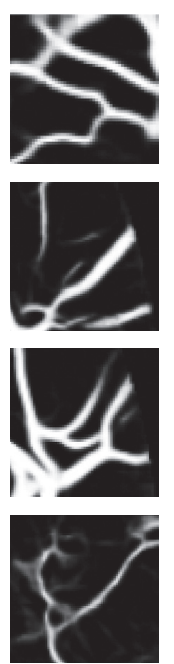

(d)
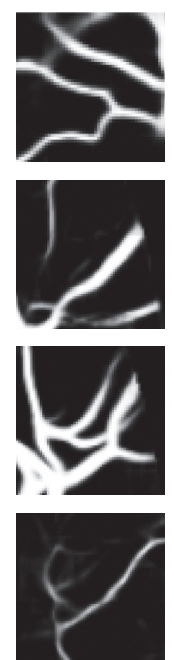

(e)
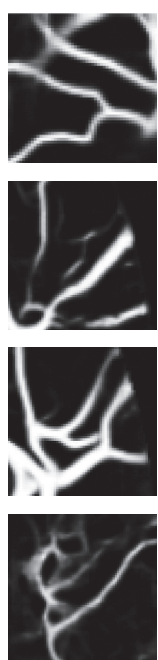

(f)
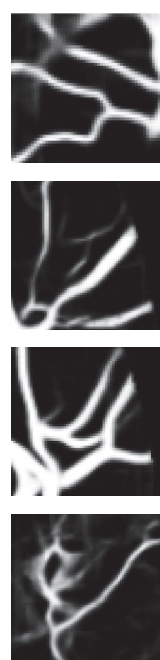

(g)
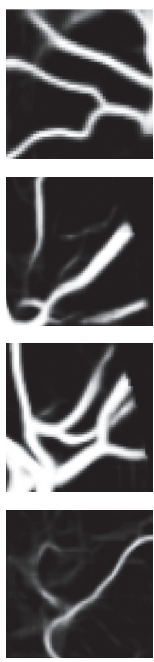

(h)

FIGURE 11: Magnified patches of different network structures on DRIVE: (a) original image; (b) magnified RGB patch; (c) ground truth; (d) Network_1; (e) Network_2; (f) Network_3; (g) Network_4; (h) WA-Net.

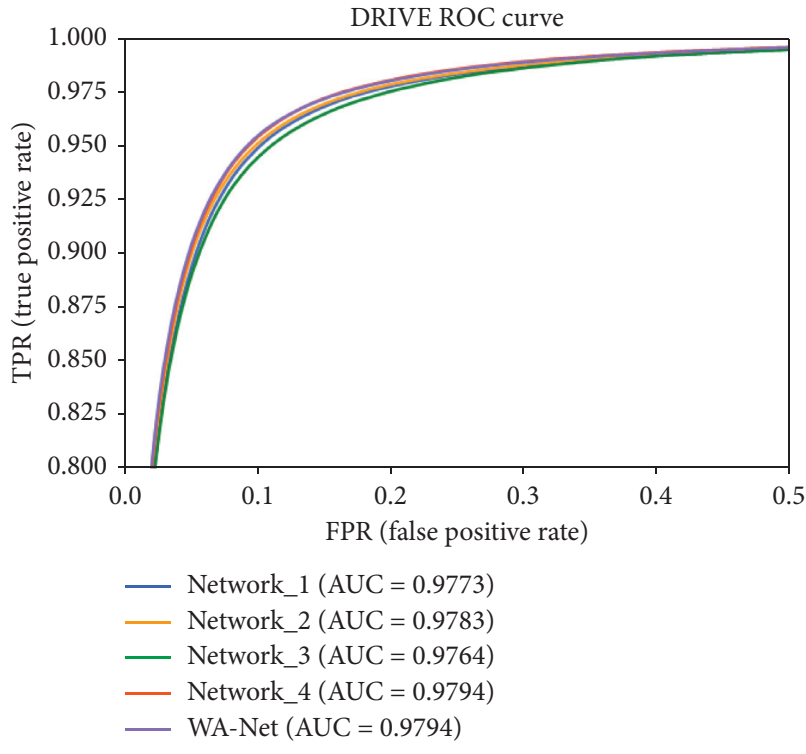

(a)

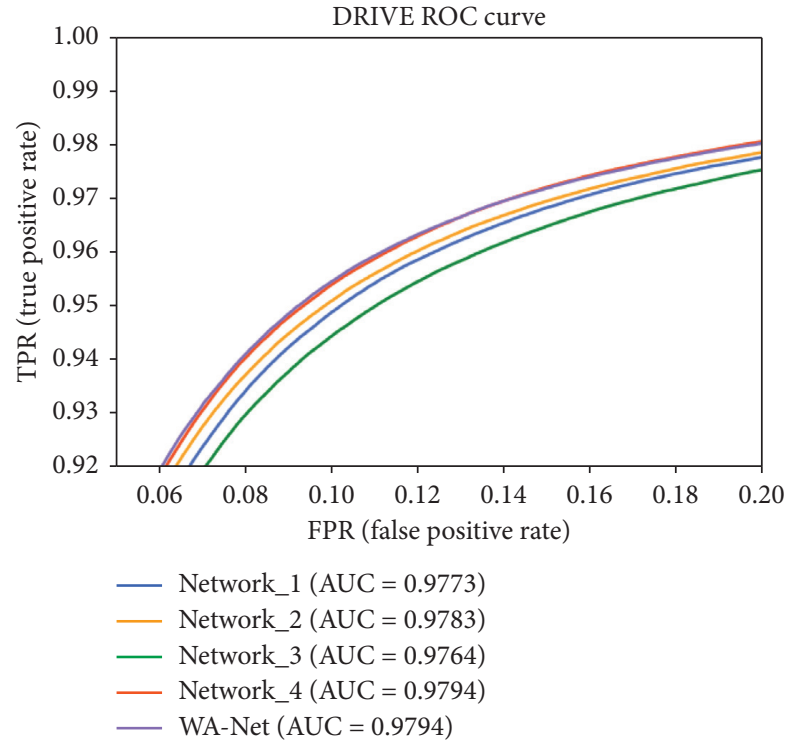

(b)

Figure 12: ROC curves of different structures on DRIVE: (a) ROC curves; (b) zoomed ROC curves on the top left of (a).

structure to three dimensions (3D) because the three-dimensional images are becoming broadly used in healthcare settings. This would be a fruitful area for further work. More than this, in the future, we can also consider adding diagnostic text to images and building new models to automatically diagnose diseases.

\section{Conclusion}

The proposed WA-Net showed excellent performance in capturing detailed blood vessels and superior performance on retinal vessel segmentation tasks with proven generalization ability. It is a general, high-performance computing framework that does not require any handcrafted features. As a consequence, it has the practical clinical application value in the automatic diagnosis system and the potential to assist doctors in the diagnosis of fundus diseases.

\section{Data Availability}

The two public open-source datasets used to support this study are available at http://www.isi.uu.nl/Research/Databases/ DRIVE/ and http://cecas.clemson.edu/ ahoover/stare/. 


\section{Conflicts of Interest}

The authors declare no conflicts of interest.

\section{Acknowledgments}

This work was supported in part by the National Natural Science Foundation of China (Grant nos. 61372023 and 62071161) and the University of Houston.

\section{References}

[1] S. You, E. Bas, D. Erdogmus, and J. Kalpathy-Cramer, "Principal curved based retinal vessel segmentation towards diagnosis of retinal diseases," in Proceedings of the IEEE First International Conference on Healthcare Informatics, Imaging and Systems Biology, pp. 331-337, San Jose, CA, USA, July 2011.

[2] M. D. Abràmoff, J. C. Folk, D. P. Han et al., "Automated analysis of retinal images for detection of referable diabetic retinopathy," JAMA Ophthalmology, vol. 131, no. 3, pp. 351-357, 2013.

[3] M. M. Fraz, P. Remagnino, A. Hoppe et al., "Blood vessel segmentation methodologies in retinal images - a survey," Computer Methods and Programs in Biomedicine, vol. 108, no. 1, pp. 407-433, 2012.

[4] H. Zolfagharnasab and A. Naghsh-Nilchi, "Cauchy based matched filter for retinal vessels detection," Journal of Medical Signals \& Sensors, vol. 4, no. 1, pp. 1-9, 2014.

[5] F. Zana and J.-C. Klein, "Segmentation of vessel-like patterns using mathematical morphology and curvature evaluation," IEEE Transactions on Image Processing, vol. 10, no. 7, pp. 1010-1019, July 2001.

[6] M. Vlachos and E. Dermatas, "Multi-scale retinal vessel segmentation using line tracking," Computerized Medical Imaging and Graphics, vol. 34, no. 3, pp. 213-227, 2010.

[7] L. Espona, M. J. Carreira, M. G. Penedo et al., "Retinal vessel tree segmentation using a deformable contour model," in Proceedings of the 19th International Conference on Pattern Recognition, pp. 1-4, Tampa, FL, USA, December 2008.

[8] Y. Hamamoto, S. Uchimura, M. Watanabe, T. Yasuda, Y. Mitani, and S. Tomita, "A gabor filter-based method for recognizing handwritten numerals," Pattern Recognition, vol. 31, no. 4, pp. 395-400, 1998.

[9] V. Nguyen and M. Blumenstein, "An application of the $2 \mathrm{~d}$ Gaussian filter for enhancing feature extraction in off-line signature verification," in Proceedings of the International Conference on Document Analysis and Recognition, pp. 339343, Ulm, Germany, August 2011.

[10] J. Staal, M. D. Abràmoff, M. Niemeijer, M. A. Viergever, and B. van Ginneken, "Ridge-based vessel segmentation in color images of the retina," IEEE Transactions on Medical Imaging, vol. 23, no. 4, pp. 501-509, 2004.

[11] E. Ricci and R. Perfetti, "Retinal blood vessel segmentation using line operators and support vector classification," IEEE Transactions on Medical Imaging, vol. 26, no. 10, pp. 13571365, 2007.

[12] X. Li, L. Wang, and E. Sung, "AdaBoost with SVM-based component classifiers," Engineering Applications of Artificial Intelligence, vol. 21, no. 5, pp. 785-795, 2008.

[13] C. Zhu, B. Zou, R. Zhao et al., "Retinal vessel segmentation in colour fundus images using Extreme Learning Machine," Computerized Medical Imaging and Graphics, vol. 55, no. 5, pp. 68-77, 2017.
[14] J. I. Orlando, E. Prokofyeva, and M. B. Blaschko, "A discriminatively trained fully connected conditional random field model for blood vessel segmentation in fundus images," IEEE Transactions on Biomedical Engineering, vol. 64, no. 1, pp. 16-27, 2017.

[15] H. Fu, Y. Xu, D. W. K. Wong et al., "Retinal vessel segmentation via deep learning network and fully-connected conditional random fields," in Proceedings of the 2016 IEEE 13th International Symposium on Biomedical Imaging, pp. 698-701, Prague, Czech Republic, April 2016.

[16] J. Mo and L. Zhang, "Multi-level deep supervised networks for retinal vessel segmentation," International Journal of Computer Assisted Radiology and Surgery, vol. 12, no. 12, pp. 2181-2193, 2017.

[17] P. Liskowski and K. Krawiec, "Segmenting retinal blood vessels with deep neural networks," IEEE Transactions on Medical Imaging, vol. 35, no. 11, pp. 2369-2380, 2016.

[18] Q. Jin, Z. Meng, T. D. Pham, Q. Chen, L. Wei, and R. Su, "DUNet: a deformable network for retinal vessel segmentation," Knowledge-based Systems, vol. 178, pp. 149-162, 2019.

[19] Z. Gu, J. Cheng, H. Fu et al., "CE-Net: context encoder network for $2 \mathrm{~d}$ medical image segmentation," IEEE Transactions on Medical Imaging, vol. 38, no. 10, pp. 2281-2292, 2019.

[20] R. Azad, M. Asadi-Aghbolaghi, M. Fathy et al., "Bi-directional convlstm u-net with densley connected convolutions," in Proceedings of the 2019 IEEE/CVF International Conference on Computer Vision Workshop (ICCVW), pp. 406-415, Seoul, Korea (South), October 2019.

[21] O. Ronneberger, P. Fischer, and T. Brox, "U-net: convolutional networks for biomedical image segmentation," in Proceedings of the International Conferences Media Image Computering Intervent. Salmon Tower Building, pp. 234-241, New York, NY, USA, March 2015.

[22] G. Huang, Z. Liu, L. Van Der Maaten et al., "Densely connected convolutional networks," in Proceedings of the 2017 IEEE Conference on Computer Vision and Pattern Recognition (CVPR), pp. 2261-2269, Honolulu, HI, USA, July 2017.

[23] H. Song, W. Wang, S. Zhao et al., "Pyramid dilated deeper convlstm for video salient object detection," in Proceedings of the European Conference on Computer Vision (ECCV), pp. 715-731, Glasgow, UK, August 2018.

[24] Ü. Budak, Z. Cömert, M. Çıbuk, and A. Şengür, "DCCMEDNet: densely connected and concatenated multi EncoderDecoder CNNs for retinal vessel extraction from fundus images," Medical Hypotheses, vol. 134, p. 109426, 2020.

[25] J. Yu, Y. Fan, J. Yang et al., "Wide activation for efficient and accurate image super-resolution," Cornell University, thaca, NY, USA, 2018, http://arxiv.org/abs/1808.08718.

[26] K. He, X. Zhang, S. Ren et al., "Deep residual learning for image recognition," in Proceedings of the 2016 IEEE Conference on Computer Vision and Pattern Recognition (CVPR), pp. 770-778, Las Vegas, NV, USA, June 2016.

[27] L.-C. Chen, G. Papandreou, I. Kokkinos, K. Murphy, and A. L. Yuille, "DeepLab: semantic image segmentation with deep convolutional nets, atrous convolution, and fully connected CRFs," IEEE Transactions on Pattern Analysis and Machine Intelligence, vol. 40, no. 4, pp. 834-848, 2018.

[28] L. C. Chen, G. Papandreou, F. Schroff et al., "Rethinking atrous convolution for semantic image segmentation," Cornell University, thaca, NY, USA, 2017, http://arxiv. org/abs/1706.05587.

[29] S. Ioffe and C. Szegedy, "Batch normalization: accelerating deep network training by reducing internal covariate shift," in 
Proceedings of the International Conference on International Conference on Machine Learning, pp. 448-456, Bejing, China, June 2015.

[30] T. Salimans and D. P. Kingma, "Weight normalization: a simple reparameterization to accelerate training of deep neural networks," in Proceedings of the Advances in Neural Information Processing Systems 29 Conference, pp. 901-909, Montreal, Canada, December 2016.

[31] F. Milletari, N. Navab, and S. Ahmadi, "V-Net: fully convolutional neural networks for volumetric medical image segmentation," in Proceedings of the 2016 Fourth International Conference on $3 D$ Vision (3DV), pp. 565-571, Stanford, CA, USA, October 2016.

[32] A. D. Hoover, V. Kouznetsova, and M. Goldbaum, "Locating blood vessels in retinal images by piecewise threshold probing of a matched filter response," IEEE Transactions on Medical Imaging, vol. 19, no. 3, pp. 203-210, 2000.

[33] A. M. Reza, "Realization of the contrast limited adaptive histogram equalization (CLAHE) for real-time image enhancement," The Journal of VLSI Signal Processing-Systems for Signal, Image, and Video Technology, vol. 38, no. 1, pp. 35-44, 2004.

[34] D. P. Kingma and J. Ba, "Adam: a method for stochastic optimization," in Proceedings of the 3rd International Conference on Learning Representations, pp. 1-15, San Diego, CA, USA, May 2015.

[35] K. He, X. Zhang, S. Ren et al., "Delving deep into rectifiers: surpassing human-level performance on ImageNet classification," in Proceedings of the IEEE International Conference on Computer Vision (ICCV), pp. 1026-1034, Venice, Italy, October 2015.

[36] C. Wang, Z. Zhao, Q. Ren, Y. Xu, and Y. Yu, "Dense U-net based on patch-based learning for retinal vessel segmentation," Entropy, vol. 21, no. 2, p. 168, 2019.

[37] S. Roychowdhury, D. D. Koozekanani, and K. K. Parhi, "Iterative vessel segmentation of fundus images," IEEE Transactions on Biomedical Engineering, vol. 62, no. 7, pp. 1738-1749, 2015.

[38] G. Azzopardi, N. Strisciuglio, M. Vento, and N. Petkov, "Trainable COSFIRE filters for vessel delineation with application to retinal images," Medical Image Analysis, vol. 19, no. 1, pp. 46-57, 2015.

[39] J. Zhang, B. Dashtbozorg, E. Bekkers, J. P. W. Pluim, R. Duits, and B. M. ter Haar Romeny, "Robust retinal vessel segmentation via locally adaptive derivative frames in orientation scores," IEEE Transactions on Medical Imaging, vol. 35, no. 12, pp. 2631-2644, 2016.

[40] Q. Li, B. Feng, and L. Xie, "A cross-modality learning approach for vessel segmentation in retinal images," IEEE Transactions on Medical Imaging, vol. 35, no. 1, pp. 109-118, 2015.

[41] Y. Chen, "A labeling-free approach to supervising deep neural networks for retinal blood vessel segmentation," Cornell University, Ithaca, NY, USA, 2017, http://arxiv.org/abs/1704. 07502.

[42] Z. Yan, X. Yang, and K.-T. Cheng, "A three-stage deep learning model for accurate retinal vessel segmentation," IEEE Journal of Biomedical and Health Informatics, vol. 23, no. 4, pp. 1427-1436, 2019.

[43] M. Z. Alom, M. Hasan, and C. Yakopcic, "Recurrent residual convolutional neural network based on u-net (r2u-net) for medical image segmentation," Cornell University, Ithaca, NY, USA, 2018, http://arxiv.org/abs/1802.06955.
[44] S. Roychowdhury, D. D. Koozekanani, and K. K. Parhi, "Blood vessel segmentation of fundus images by major vessel extraction and subimage classification," IEEE Journal of Biomedical and Health Informatics, vol. 19, no. 3, pp. 11181128, 2015.

[45] Z. Yan, X. Yang, and K.-T. Cheng, "Joint segment-level and pixel-wise losses for deep learning based retinal vessel segmentation," IEEE Transactions on Biomedical Engineering, vol. 65, no. 9, pp. 1912-1923, 2018.

[46] K. He, X. Zhang, S. Ren, and J. Sun, "Identity mappings in deep residual networks," in Proceedings of the Computer Vision-ECCV 2016', European Conference on Computer Vision, pp. 630-645, Amsterdam, The Netherlands, October 2016. 